Formación Universitaria

Vol. 7(1), 3-12 (2014)

doi: $10.4067 / S 0718-50062014000100002$

\title{
Evaluación de Impactos Ambientales Bióticos en la Industria Química Mediante uso de Software Libre
}

\author{
Fernando Gutiérrez-Martín ${ }^{(1)}$, Antonio Nieto-Márquez ${ }^{(1)}$, Evangelina Atanes ${ }^{(1)}$ y Mercedes Ruiz- \\ Pastrana $^{(2)}$ \\ (1) Universidad Politécnica de Madrid, Dept. Química Industrial y Polímeros. EUITI. Rda Valencia 3. E-28012 Madrid- \\ España (e-mail: fernando.gutierrez@upm.es; antonio.nieto@upm.es; evangelina.atanes@upm.es) \\ (2) Universidad de Valladolid, Dept. Didáctica CC. Experimentales. FEYTS. Campus M. Delibes. E-47011 Valladolid- \\ España (e-mail: pastrana@dce.uva.es)
}

Recibido Jun. 4, 2013; Aceptado Jul. 26, 2013; Versión final recibida Sep. 3, 2013

\begin{abstract}
Resumen
Se presenta el desarrollo de los aspectos metodológicos necesarios para evaluar los riesgos asociados a la síntesis de productos químicos. El trabajo forma parte de los esfuerzos que vienen realizándose en las universidades Politécnica de Madrid y de Valladolid en España por introducir los componentes ambientales dentro de los planes docentes de las ciencias y las tecnologías. Como caso de estudio se plantea el análisis de rutas alternativas y del riesgo tóxico asociado a la síntesis de 1-naftil-metilcarbamato. Se usa software libre (EPI Suite y Mackay Level III) y diversas bases de datos. La importancia del estudio para la formación de profesionales es el uso de criterios sistemáticos con base en la química y la evaluación ambiental. Se concluye que el uso de los programas y métodos descritos constituyen herramientas para valorar e interpretar los impactos bióticos de un proceso tanto en la etapa formativa como en la vida profesional.
\end{abstract}

Palabras clave: evaluación de impacto ambiental, riesgo químico, potencial de toxicidad, software libre, procesos químicos

\section{Evaluation of Biotic Environmental Impacts in Chemical Process Industries using Free Software}

\begin{abstract}
The methodological aspects required for evaluating the risks associated to the synthesis of chemical products is presented. The study is part of the efforts done at the universities Polytechnic of Madrid and of Valladolid in Spain. Chemical risk assessment, such as estimating the inputs of contaminants, their environmental fates and impact indexes, are presented. These concepts are illustrated by means of a case study where the biotic impacts from alternative reaction pathways to produce 1-naftil-metilcarbamato are analyzed using the EPI Suite and Mackay Level III software, together with proper databases. Its relevance in higher education is mainly related to the use of systematic criteria based in chemistry and environmental valuations. It is concluded that the use of the method and program described are valuable tools to interpret biotic impact both during the professional formation and during the professional life.
\end{abstract}

Keywords: environmental impact assessment, chemical risk, toxicity potential, free software, chemical process industries 


\section{INTRODUCCION}

Tradicionalmente, los Ingenieros Químicos han enfocado el diseño de sus procesos hacia la optimización de los mismos en términos fundamentalmente económicos. Sin embargo, debido a la problemática asociada a la síntesis y utilización de productos químicos, tanto en procesos industriales como en el consumo, y sus consecuencias sobre el medio ambiente, surge la necesidad de evaluar e interpretar los impactos producidos (Alha et al., 2000; Gutiérrez-Martín y Dahab, 1998; Gutiérrez-Martín y Hüttenhain, 2003; Mulder, 2006).

Para cumplir con la cada vez más estricta legislación ambiental, se utilizan tecnologías de fin de línea, tales como filtros, lavadores, incineradoras y otros sistemas de depuración. Esta solución, sin embargo, debería ser la última dentro del paradigma de la producción limpia, dando prioridad a los procesos que desde su diseño tengan un menor impacto. Puesto que un proceso químico bien diseñado es, a su vez, un proceso con bajo impacto ambiental (i.e. reducción de subproductos, integración energética, etc.), deben primarse las acciones preventivas sobre las correctivas, buscando rutas de síntesis más limpias, selección de reactivos menos contaminantes o incluso plantearse el uso de otros productos que satisfagan las mismas necesidades (Lemkowitz et al., 1996; Allen y Rosselot, 1997; Gutiérrez et al., 2003; García-Serna et al., 2007).

En esta apuesta por el diseño de procesos y/o productos más respetuosos con el medio ambiente, resulta de gran utilidad el uso de herramientas informáticas que permitan evaluar de manera cuantitativa el impacto ambiental de las diferentes alternativas a la hora de diseñar un proceso/producto. Existen diversos recursos web de acceso libre que permiten la estimación de las propiedades fisicoquímicas, la toxicidad y distribución ambiental de un amplio catálogo de productos químicos. A diferencia de otros programas de simulación, con licencias costosas y acceso limitado, estos programas ofrecen a los alumnos recursos accesibles, tanto en el centro universitario como en sus hogares, para el estudio de los impactos ambientales; por ello, constituyen materiales didácticos de especial interés para las universidades, a la vez que tienen aplicación en el mundo profesional de las ingenierías (Allen y Shonnard, 2002; Gutiérrez y Quirce, 2004; Serrano y Narváez, 2010).

La mayoría de estos recursos han sido desarrollados en Norteamérica (EEUU y Canadá), por lo que su uso en Europa o en los países Latinoamericanos está poco extendido y en muchos casos son desconocidos por la comunidad académica o profesional. Por ello, apenas hay referencias relevantes en este ámbito al menos en artículos publicados en revistas de corriente principal (Gutiérrez-Martín et al., 2007a). Este trabajo forma parte de los esfuerzos que vienen realizándose en nuestros departamentos por introducir los componentes ambientales dentro de los planes docentes universitarios de las ciencias y las tecnologías, ampliando resultados parciales que han sido presentados previamente en congresos relevantes sobre estas materias (OCW, 2006; Gutiérrez-Martín et al., 2007b; Nieto-Márquez et al., 2012a, 2012b). Como ejemplo, se plantea un análisis de rutas de síntesis alternativas y del riesgo tóxico asociado a los diferentes reactivos/productos químicos implicados.

\section{EVALUACION AMBIENTAL DE SUSTANCIAS QUIMICAS}

La evaluación de riesgos ambientales requiere de la aplicación de herramientas sistemáticas, que combinen la estimación de los flujos de descarga (emisiones), el transporte y destino de los contaminantes en los medios receptores (niveles de inmisión), y sus efectos potenciales sobre la salud y los ecosistemas (índices de impacto). Estos efectos se manifiestan a distintas escalas espacio-temporales y abarcan tanto categorías bióticas (toxicidad en los organismos vivos), como abióticas (cambio climático, capa de ozono, acidificación, eutrofización, niebla fotoquímica, residuos, etc.), y de sostenibilidad económica (agotamiento de recursos). La figura 1 ilustra un escenario generalizado de la cadena de causas-efectos relacionados con las descargas tóxicas de una industria química (emisiones aéreas, efluentes acuosos y residuos sólidos), donde el riesgo sobre la salud asociado con un contaminante depende del flujo de dicha sustancia, su destino ambiental, la exposición humana y la respuesta biológica: la fuente de origen determina la naturaleza de las sustancias, su flujo de descarga y el medio donde son vertidas; una vez introducidos en el medio, los contaminantes se dispersan asociándose a las distintas fases del mismo, según su afinidad, a la vez que pueden tener lugar reacciones químicas que los transformen.

La evaluación comienza con la identificación de las fuentes y descargas, sus concentraciones en sustancias tóxicas y características de la matriz (gas, líquida, o sólida), para estimar las cantidades liberadas al aire, las aguas o el suelo; entonces se modela el transporte y transformación de los contaminantes a través de estos medios, junto con su asimilación por inhalación, ingestión o contacto dérmico, para determinar la cantidad de los agentes tóxicos que llegan a afectar a las poblaciones. 


\section{Cuantificación de las descargas ambientales}

Los objetivos del análisis determinarán el tipo y detalle de la información precisa en cada caso; a partir del diagrama de flujo, puede identificarse las corrientes de salida del proceso que no resultan productos útiles (descargas potenciales); determinadas las fuentes de descarga, deben estimarse sus cantidades: cuando no se dispone de medidas directas (en la fase de diseño), deben emplearse factores o modelos de emisión.

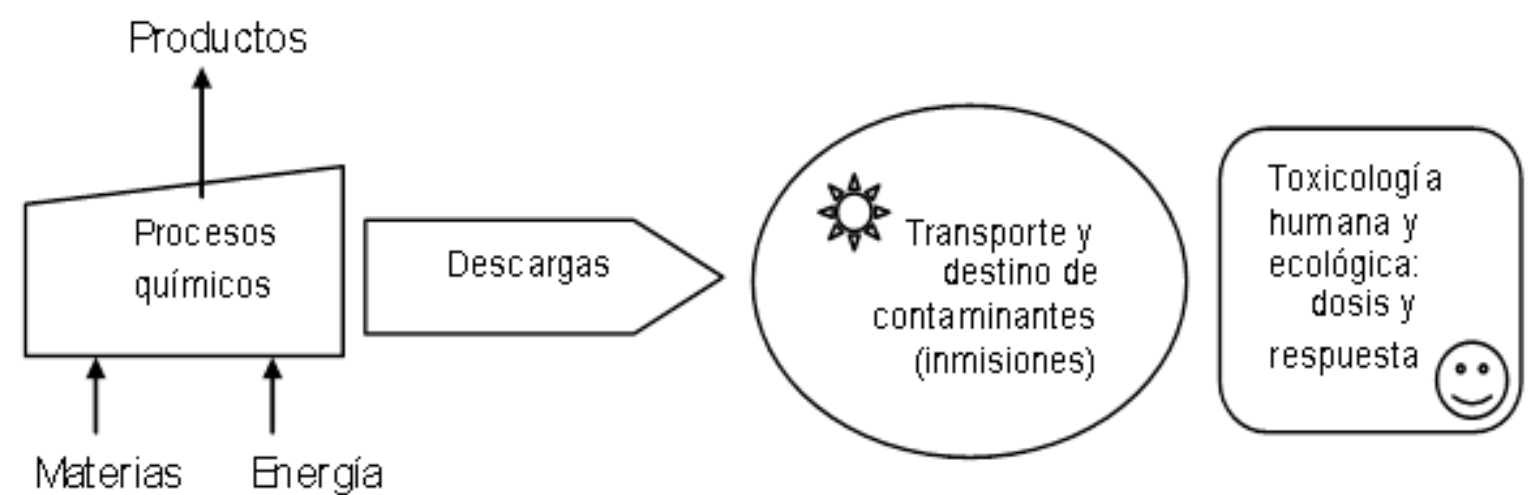

primas Medio ambiente

Fig. 1: Descargas tóxicas y cadena de efectos

\section{Comportamiento y destino de los contaminantes}

Después de que un contaminante se libera en alguno de los compartimentos ambientales (atmósfera, aguas superficiales o suelos), existen diversos procesos de transporte y reacción que afectan a su concentración final en dichos medios. Para describirlos deben conocerse los parámetros fisicoquímicos de la sustancia y el medio que determinan la naturaleza/velocidad del transporte, la distribución entre fases y la cinética química (Mackay y Paterson, 1991; Mackay, 2001; Howard y Meylan, 2001; Rosenbaum et al., 2007).

Uno de los métodos aplicables es el modelo de fugacidades de Mackay, que predice las concentraciones de estado estacionario de la sustancia en el aire [1], agua [2], suelo [3] y sedimentos [4], como respuesta a su emisión constante en una región definida $\left(E_{i}\right)$; la figura 2 resume los parámetros de transporte por advección desde y hacia las zonas limítrofes aéreas o acuáticas $\left(G_{A i} C_{B i}, D_{A i}\right)$, los coeficientes de transferencia de masa intermedia $\left(D_{i j}\right)$, así como las reacciones de oxidación, hidrólisis, fotólisis y biológicas en cada región $\left(D_{\mathrm{Ri}}\right)$. Su solución es muy compleja, pero pueden utilizarse hojas de cálculo y el programa de software disponible en la web (CEMC, 2011).

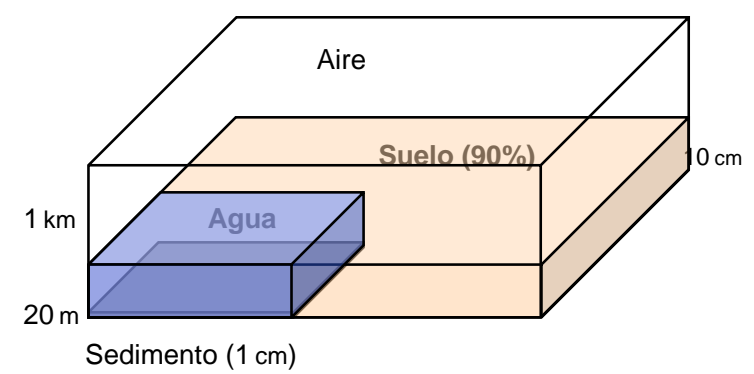

Dominio del modelo $\left(10^{5} \mathrm{~km}^{2}\right)$
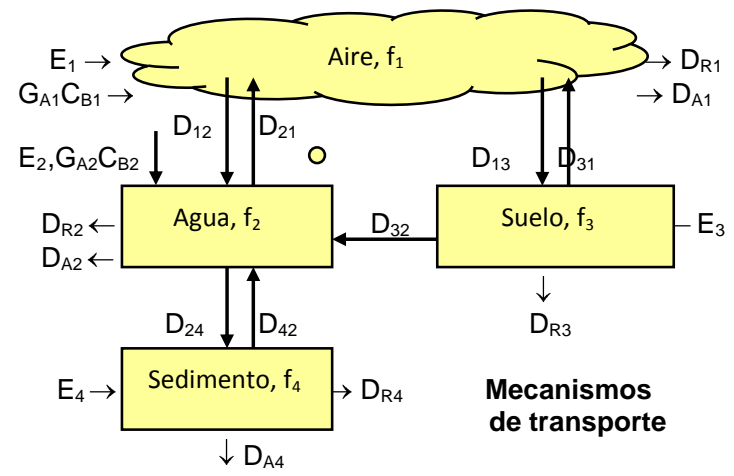

Mecanismos

de transporte

Fig. 2: Modelo ambiental multimedia de fugacidades (Mackay Level III) 
Esta clase de riesgos se definen como la probabilidad de que ocurra un peligro por exposición a un agente químico (Gutiérrez y Domínguez, 2006); para caracterizarlos se debe conocer la cantidad de contaminante en los individuos (exposición), así como su potencial para causar un daño o una lesión (toxicidad).

Riesgo químico $\left(\mathrm{I}_{\mathrm{i}}\right)=\mathrm{f}($ exposición, toxicidad $)$

Los índices de riesgo utilizados para la métrica ambiental, pueden expresarse como producto del potencial inherente de toxicidad (IP) y la exposición (EP), en forma relativa (adimensional), i.e. tomando un compuesto como referencia $(r)$ y sumando las emisiones pertinentes $(\mathrm{mi})$ para cada categoría de impacto:

$$
\mathrm{l}_{\text {ir }}=(E P \cdot I P)_{i} /(E P \cdot I P)_{r} \quad I_{r}=\Sigma m_{i} \cdot l_{i r}
$$

Puede distinguirse el potencial de toxicidad para efectos carcinógenos y no cancerígenos sobre la salud, así como según las rutas de exposición predominantes (inhalación e ingestión). La toxicidad no cancerígena en humanos está controlada por exposiciones umbral, por lo que los parámetros pueden ser concentraciones o dosis de referencia [RfC mg/m ${ }^{3}, \mathrm{RfD} \mathrm{mg} / \mathrm{kg} \cdot$ día], valores letales para el $50 \%$ de los organismos $\left(\mathrm{LC}_{50}, \mathrm{LD}_{50}\right)$, u otros límites umbral, permisibles o recomendados [TLVs, PELs, RELs].

En el caso de los carcinógenos se emplea como parámetro el factor de pendiente (SF, $\mathrm{kg} \cdot \mathrm{dí} / \mathrm{mg}$ ), que puede obtenerse mediante ensayos dosis-respuesta con animales (i.e. exceso de cánceres vs dosis administrada); también se utilizan tabulaciones de expertos basadas en la evidencia [WOE], junto a un valor de riesgo [HV] (NIHS, 2006; EPA, 2008; NTP, 2013; OSHA, 2013).

Los índices de riesgo se basan en las concentraciones estacionarias de contaminantes en aire o agua $\left(\mathrm{C}_{\mathrm{i}, \mathrm{m}}\right)$, tras una descarga de $1000 \mathrm{~kg} / \mathrm{h}$ (estimadas con el modelo multimedia de Mackay); dichas concentraciones se convierten en dosis mediante factores de asimilación estándar $\left(\mathrm{u}=20 \mathrm{~m}_{\text {aire }}^{3} /\right.$ día para inhalación y $2 \mathrm{l}_{\text {agua }} /$ día para ingestión oral, y una masa corporal $p=70 \mathrm{~kg}$ ):

$D_{i}=\left(C_{i, m} \cdot u\right) / p$

Finalmente, las dosis se transforman en riesgo con los parámetros de toxicidad anteriores, y los índices se obtienen por comparación con un compuesto de referencia en cada caso (tolueno y benceno).

Para peligros no cancerígenos, las dosis se dividen por las de referencia para determinar el riesgo tóxico, se definen los potenciales de toxicidad por inhalación o ingestión (INHTP, INGTP) en relación al tolueno, y se agregan los efectos de todos los contaminantes, multiplicados por sus flujos de descarga, para obtener un índice global del proceso:

$$
\mathrm{TP}_{\mathrm{i}}=\left(\mathrm{C}_{\mathrm{i}, \mathrm{m}} / \mathrm{RfX}_{\mathrm{i}}\right) /\left(\mathrm{C}_{\mathrm{T}, \mathrm{m}} / \mathrm{RfX}_{\mathrm{T}}\right) \quad \mathrm{I}_{\mathrm{T}}=\Sigma \mathrm{m}_{\mathrm{i}} \cdot \mathrm{TP}_{\mathrm{i}}
$$

Para 'cancerígenos', los índices de toxicidad para ambas rutas de exposición se obtienen de modo similar, empleando ahora los factores de pendiente disponibles (o valores de riesgo HV en su caso), con el benceno como referencia:

$$
\mathrm{CP}_{\mathrm{i}}=\left(\mathrm{C}_{\mathrm{i}, \mathrm{m}} \cdot \mathrm{SF}_{\mathrm{i}}\right) /\left(\mathrm{C}_{\mathrm{B}, \mathrm{m}} \cdot \mathrm{SF}_{\mathrm{B}}\right) \quad \mathrm{I}_{\mathrm{C}}=\Sigma \mathrm{m}_{\mathrm{i}} \cdot \mathrm{CP}_{\mathrm{i}}
$$

La caracterización de riesgos resulta pues de conjugar toda la información disponible, incluyendo los peligros potenciales, las rutas y magnitud de la exposición, y la población afectada; en caso de que intervengan varias sustancias o vías de incidencia, debe considerarse su efecto agregado y acumulativo.

Este tipo de información es de gran utilidad para evaluar la necesidad de tomar medidas para la protección de los ecosistemas respecto a la acción de los contaminantes. El paso final es la gestión de riesgos, referida a la comunicación a la población afectada, la consideración de opciones, el control de las decisiones y el monitoreo; los objetivos son identificar, evaluar, seleccionar e implementar las correspondientes medidas de reducción (las respuestas dependerán en general de una serie de elementos culturales y escalas de valores); en cualquier caso, es evidente que la minimización de los riesgos a través del desarrollo y diseño proactivo de los procesos debe situarse en el corazón de las prácticas ingenieriles.

\section{RESULTADOS DEL CASO DE ESTUDIO}




\section{Rutas de síntesis del 1-naftil metilcarbamato (carbaryl)}

En diciembre de 1984 se produjo en Bophal (India) un desastre químico de proporciones jamás conocidas con más de tres mil muertos y cincuenta mil supervivientes seriamente afectados. El producto fabricado en esta población era el insecticida carbaryl que implica una reacción química bastante simple, pero con dos precursores muy peligrosos como son el fosgeno e isocianato de metilo (MIC):

$\mathrm{CH}_{3} \mathrm{NH}_{2}+\mathrm{COCl}_{2} \rightarrow \mathrm{CH}_{3} \mathrm{NCO}-$ naftol $\left(\mathrm{C}_{10} \mathrm{H}_{7} \mathrm{OH}\right) \rightarrow \mathrm{C}_{10} \mathrm{H}_{7} \mathrm{OCONHCH}$

La causa inmediata del accidente fue la introducción de un gran volumen de agua en un depósito con 60 ton de MIC, durante una operación rutinaria de mantenimiento; ello causó un gran aumento de la temperatura y la presión, que llevó a la explosión del tanque de almacenamiento y la formación de una nube tóxica de MIC y sus productos de hidrólisis, como el cianuro de hidrógeno, que se dispersaron sobre la población cercana.

Es fácil encontrar culpables entre los operarios, procedimientos o fallos de los equipos en casos como este, aunque podrán volver a ocurrir ya que los humanos están sujetos a errores, los protocolos siempre pueden mejorarse echando la vista atrás, y hasta el mejor dispositivo ingenieril puede en algunas ocasiones fallar. Por ello, es mejor identificar la raíz del problema para tratar de evitarlo en origen: en este caso, la causa fue el gran inventario de MIC, por lo que deberíamos preguntarnos si es posible producir carbaryl eficazmente sin necesidad de usar dicha sustancia y responder a ello en las primeras fases del diseño (Lancaster, 2002).

En términos simples, hemos visto que el riesgo químico es función de la exposición y peligros, por lo que ha venido controlándose de modo convencional limitando la exposición vía equipos de protección, elementos de seguridad y métodos de control; por contra, el 'diseño inherentemente seguro' pone su acento en el peligro potencial como factor a minimizar, lo que constituye una alternativa mucho más radical y efectiva. En este caso, el fosgeno podría obtenerse in-situ ('just in time') y evitar el uso de MIC por reacción inicial de fosgeno con naftol a cloro-formiato y posterior reacción de este con metil-amina.

No obstante, las herramientas sistémicas cuantitativas para identificar alternativas descansan en la química combinatoria: la primera etapa es la selección de un conjunto de reactivos con bases químicas, y después aplicar restricciones para deducir aquellas que sean posibles (mediante la estequiometría y termodinámica); finalmente, las reacciones se valoran siguiendo criterios económicos y ambientales. Por ejemplo, para la síntesis del 1-naftil metilcarbamato se ha empleado 1-naftol e isocianato de metilo intermedio, pero pueden identificarse un conjunto de grupos relacionados con la molécula como base para la selección de reactivos potenciales $\left(\mathrm{C}_{6} \mathrm{H}_{5}, \mathrm{CH}_{3} \mathrm{NH}, \mathrm{COO}, \mathrm{CHO}\right.$, etc.); así podrían plantearse 13 reacciones y 15 especies: [1] carbaryl, [2] oxígeno, [3] hidrógeno, [4] cloruro de hidrógeno, [5] metil-formamida, [6] agua, [7] metil-amina, [8] fosgeno, [9] metil-isocianato, [10] naftol, [11] naftaleno, [12] cloro-naftaleno, [13] cloro-metano, [14] metanol, y [15] clorometanal (Tabla 1).

Tabla 1: Rutas de síntesis, reactivos y coeficientes estequiométricos para la síntesis de carbaryl

\begin{tabular}{|c|c|c|c|c|c|c|c|c|c|c|c|c|c|c|c|}
\hline Ruta / reactivo & 1 & 2 & 3 & 4 & 5 & 6 & 7 & 8 & 9 & 10 & 11 & 12 & 13 & 14 & 15 \\
\hline 1 & 1 & -1 & & & & & & & -1 & 1 & -2 & & & & \\
\hline 2 & 1 & & 1 & 1 & -1 & -1 & & & & & & -1 & & \\
\hline 3 & 1 & & 2 & & -1 & -1 & & & & & -1 & & & & \\
\hline 4 & 1 & & 1 & & -1 & & & & & & & -1 & 1 & -1 & \\
\hline 5 & 1 & -1 & & & -1 & 1 & & & & & -1 & & & & \\
\hline 6 & 1 & & & 1 & & -1 & & & -1 & & & -1 & & & \\
\hline 7 & 1 & & & & -1 & & & & & & -1 & & 1 & & -1 \\
\hline 8 & 1 & & 1 & & & -1 & & & -1 & & -1 & & & & \\
\hline 9 & 1 & & & & & & & & -1 & & & -1 & 1 & -1 & \\
\hline 10 & 1 & & & 2 & & & -1 & -1 & & -1 & & & & \\
\hline 11 & 1 & & 1 & 1 & & & -1 & & & -1 & & & & -1 \\
\hline 12 & 1 & 1 & & & -1 & & & & & -1 & & & & \\
\hline 13 & 1 & & & & & & & & -1 & -1 & & & & \\
\hline
\end{tabular}


En este apartado, evaluaremos los índices de impacto de dos de las rutas de síntesis seleccionadas en el anterior: a partir de naftol (NPO) e isocianato de metilo (MIC), o con naftaleno (NPE) y metil-formamida (MFA), utilizando los balances estequiométricos teóricos, así como las propiedades de distribución, persistencia y toxicidad de las sustancias implicadas:

Ruta 13: $1 \mathrm{NPO}+1 \mathrm{MIC}=1$ Producto

Ruta 5: $1 \mathrm{NPE}+1 \mathrm{MFA}=1$ Producto

La tabla 2 detalla las propiedades de las sustancias relevantes para el cálculo de la exposición y toxicidad, obtenidas con el programa EPI Suite (a través de su base de datos y contribuciones de grupo) (EPA, 2012), así como los valores límite umbral para estos compuestos o subrogados ( ${ }^{1}$ fenol, ${ }^{2}$ formamida) (ACGHI, 2011). En la figura 3 se muestra la pantalla principal del programa desde la que puede accederse a la base de datos experimentales y al cálculo de las propiedades deseadas, utilizando como ejemplo el isocianato (MIC).

Tabla 2: Propiedades de distribución, persistencia y toxicidad de las sustancias químicas empleadas

\begin{tabular}{|c|c|c|c|c|}
\hline & & & & \\
\hline & NPO & MIC & NPE & MFA \\
\hline Peso molecular, $\mathrm{g} / \mathrm{mol}$ & 144,17 & 57,05 & 128,18 & 59,07 \\
\hline Solubilidad en agua, mg/l & 866 & $3,276 \cdot 10^{4}$ & 31 & $1 \cdot 10^{6}$ \\
\hline Presión de vapor, $\mathrm{Pa}\left(25^{\circ} \mathrm{C}\right)$ & 0,0365 & $4,64 \cdot 10^{4}$ & 11,33 & 33,7 \\
\hline Coeficiente de partición, Ig $\mathrm{K}_{\mathrm{ow}}$ & 2,85 & 0,79 & 3.30 & $-0,97$ \\
\hline Punto de fusión, ${ }^{\circ} \mathrm{C}$ & 95 & -45 & 80,2 & $-3,8$ \\
\hline Vidas medias: aire & 0,467 & 1890 & 11,9 & 37,9 \\
\hline agua & 360 & 360 & 900 & 360 \\
\hline \multirow{2}{*}{$\begin{array}{l}\text { suelo } \\
\text { sedimento }\end{array}$} & 720 & 720 & 1800 & 720 \\
\hline & 3240 & 3240 & 8100 & 3240 \\
\hline Umbral de riesgo (TWA), ppm & ${ }^{1} 5$ & 0,02 & 2 & ${ }^{2} 10$ \\
\hline
\end{tabular}

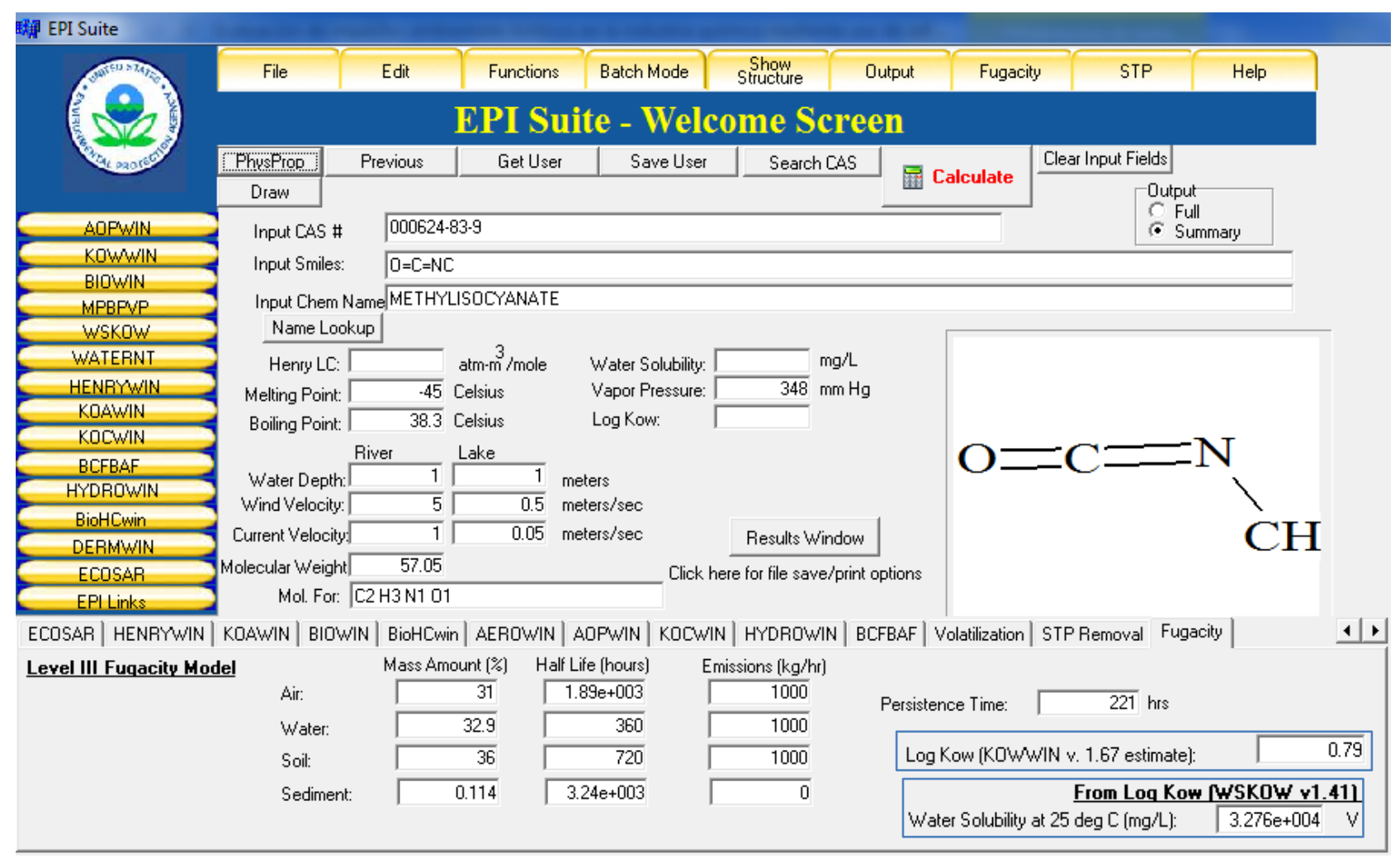

Fig. 3: Interfaz de estimación de propiedades físico-químicas y ambientales (EPISuite)

La figura 4 muestra los resultados de distribución y concentración estacionaria de isocianato de metilo en los diferentes compartimentos ambientales para una emisión aérea de $1000 \mathrm{~kg} / \mathrm{h}$ en el medio ambiente estándar 
del programa Mackay L-III. La tabla 3 resume finalmente los resultados de inmisión en el medio atmosférico para todas las sustancias implicadas, los cocientes de riesgo no cancerígeno basados en la concentración tóxica (TWA), así como los índices de toxicidad combinados de ambas rutas de síntesis para una descarga equivalente al consumo de reactivos teórico por mol de producto (ec. 4).

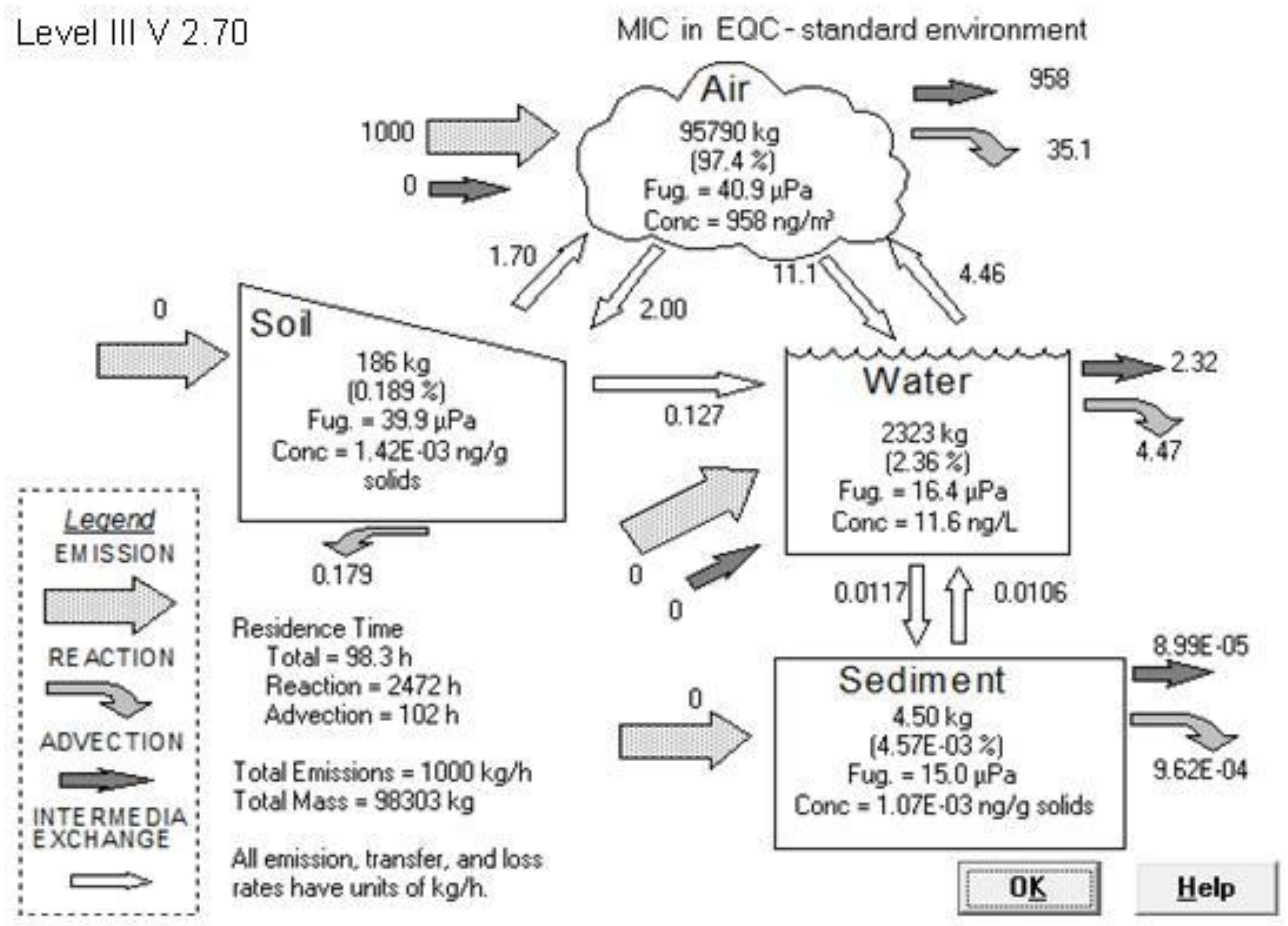

Fig. 4: Resultados del programa multimedia de fugacidades (Mackay Level III)

Tabla 3: Resultados de exposición, cocientes de riesgo e índices de impacto para las rutas de síntesis

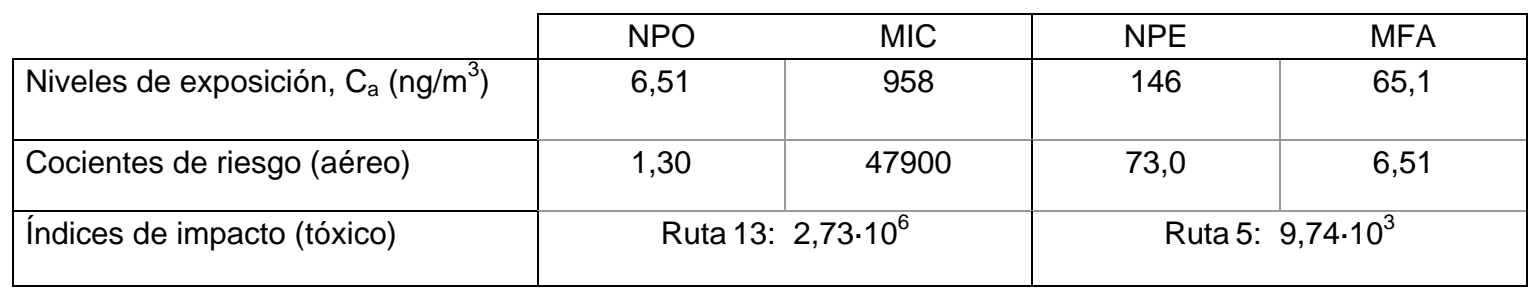

\section{DISCUSIÓN}

Se ha presentado un método sistemático para la evaluación de los impactos bióticos de sustancias químicas, donde los efectos dependen de la dosis, y ésta es función de la concentración, que a su vez se relaciona con los flujos de descarga: por tanto, las emisiones son el punto de partida; la concentración en cada subsistema se relaciona con las propiedades fisicoquímicas y ambientales (modelos de destino de los contaminantes); finalmente, la dosis-concentración se convierte en índice de riesgo con datos de toxicidad (riesgo inherente). El resultado de la evaluación es un conjunto de índices (métrica ambiental), representativos de los impactos potenciales de los procesos para la salud humana y/o ecológica; los índices pueden utilizarse para objetivos de diseño como ranking de tecnologías, optimización de procesos, operación de reactores químicos, etc.

Como se observa en el caso estudiado, ambas rutas de síntesis del producto presentan índices de impacto potencial muy diferentes, lo que constituye un criterio relevante para seleccionar las alternativas del proceso. No obstante, aunque los resultados son muy sugestivos, no puede afirmarse que este análisis sea siempre la panacea para obtener la ruta reactiva óptima con total certidumbre; su principal interés está en el uso de criterios de decisión sistemáticos para la búsqueda de buenos materiales de partida con base en la química estequiométrica, la termodinámica, y las evaluaciones ambiental y económica, que bien pueden conducir a la alternativa deseable o más simplemente a una clarificación de las restricciones existentes en su desarrollo. En nuestro caso, el análisis se ha limitado a los impactos tóxicos por vía respiratoria, que requieren datos de toxicidad por inhalación, los cuales son a veces limitados (por ello se toman subrogados del NPO y MFA). 
En general, deben rastrearse las bases de datos IRIS (EPA), el 'Sistema Experto para la Química Verde' (GCES), la página web ECO-informas (ISTAS), las fichas de productos químicos para valores ambientales y de seguridad, recomendaciones y alternativas, etc. (EPA, 2002; EPA, 2013; ISTAS, 2006).

Entre todos los recursos informativos y analíticos, cabe destacar RISCTOX, la base de datos ISTAS, como herramienta única en el mundo y en idioma Castellano para la comunidad hispanohablante. Así, J. Tickner, director del Centro Lowell de Producción Sostenible de la Universidad de Massachusetts (EEUU), afirma que "no hay otra base de datos que contenga 100.000 sustancias químicas tóxicas con alternativas de sustitución, que tenga tan claro su enfoque en prevención, que sea tan fácil de usar, que contenga tantos casos prácticos; lo peor de RISCTOX es que internacionalmente no sea más conocida; la he mostrado a colegas de EEUU y se han quedado impresionados" (Chulvi, 2010).

Cabe añadir que pueden también utilizarse las aproximaciones de grupo para diseñar rutas y productos más benignos, como las relaciones estructura-reactividad (SAR), para identificar las funciones tóxicas o convertir sus destinos en más inocuos; las SAR pueden servir para predecir modificaciones que mejoren la seguridad ambiental de una molécula; también pueden eliminarse o enmascararse los grupos tóxicos que definan una clase de compuestos (e.g. aldehídos, nitrilos, isocianatos); si todo esto no resulta posible, puede actuarse sobre otras propiedades como pueden ser la biodisponibilidad (piel, pulmón, intestino), o la persistencia en el medio ambiente (productos degradables tras su vida útil). Así, el diseño ambiental de las sustancias no se limita solamente a los peligros durante sus etapas de fabricación y uso, sino también después del vertido y final del ciclo de vida.

En general, deben rastrearse cuidadosamente páginas web como las indicadas, bases de datos específicas, fichas de seguridad, sistemas expertos, correlaciones de grupo, métodos heurísticos para alternativas, etc. Por otro lado, la selección final debe considerar la factibilidad técnica, social y económica del proceso, junto a los impactos sobre la seguridad, la salud y el medio ambiente en su conjunto.

Finalmente, el interés que suscita la utilización de esta metodología, datos y programas informáticos entre los alumnos de ingeniería es sin duda de gran relevancia en el contexto de la formación universitaria integral, ya que permiten englobar conocimientos y aplicarlos en la toma de decisiones que sitúan la variable ambiental en el centro del pensamiento y las aplicaciones tecnológicas. En la actualidad hay disponible software libre muy potente, interesante y de fácil uso, que nuestros alumnos, en las universidades Politécnica de Madrid y de Valladolid en España, llevan utilizando durante muchos cursos, con los buenos resultados que ahora deseamos compartir por este medio de comunicación académica.

\section{CONCLUSIONES}

De acuerdo a lo presentado en la metodología, el caso de estudio y la discusión, se destacan las siguientes conclusiones:

1.- La evaluación de riesgos químicos requiere aplicar herramientas que combinan los flujos de descarga, el destino de los contaminantes y sus efectos potenciales; los índices así obtenidos pueden utilizarse para la selección de tecnologías, diseño de procesos, etc.

2.- En nuestro caso, las rutas de síntesis estudiadas presentan índices de impacto potencial muy diferentes, lo que constituye un criterio relevante para seleccionar las alternativas del proceso con base en la química y la evaluación ambiental sistemática de los materiales de partida.

3.- El uso de los programas y métodos descritos en este trabajo no solo constituyen herramientas poderosas para valorar e interpretar los impactos bióticos producidos en un determinado proceso, sino que permiten a los estudiosos disponer de estos recursos de un modo fácilmente accesible en cualquier lugar, tanto durante la etapa formativa como en el futuro profesional.

\section{AGRADECIMIENTOS}

A la Universidad Politécnica de Madrid (UPM) por su apoyo y financiación del Proyecto de Innovación Educativa "Análisis para la implantación y desarrollo de nuevos procesos educativos en la EUIT Industrial" (2005-06), dentro del cual se abordaron y pusieron a punto muchos de los conceptos y métodos descritos en el presente trabajo. 


\section{REFERENCIAS}

ACGHI, Threshold Limit Values for Chemical Substances, Physical Agents and Biological Exposure Indices (TLV \& BEIs). American Conference of Governmental Industrial Hygienists, Cincinnati, OH, USA (2011).

Alha K., C. Hollinger, B.S. Larsen, P. Purcell y W. Rauch, Environmental engineering education: summary report of the $1^{\text {st }}$ European Seminar, Water Science and Technology, 2, 1-7 (2000).

Allen, D.T., Rosselot, K.S., Pollution Prevention for Chemical Processes. Wiley Pub., New York, USA (1997).

Allen, D.T., Shonnard, D.R., Green Engineering: Environmentally Conscious Design of Chemical Processes. Prentice Hall PTR Publishers, Upper Saddle River, NJ, USA (2002).

CEMC, Level III Model, v 2.70 (en línea). Centre for Environmental Modeling \& Chemistry, Trent University, Peterborough, Ontario, Canada (2011). http://www.trentu.ca/academic/aminss/envmodel/models/VBL3.html. Acceso: 18 de Abril (2013).

Chulvi, B., Entrevista a Joel Tickner "Las empresas necesitan apoyo de la ciencia para sustituir los tóxicos", Por Experiencia, 48 (2010)

EPA. Green Chemistry Expert System (en línea). US Environmental Protection Agency, Washington, DC, USA (2002). http://www.epa.gov/greenchemistry/, http://fusion.stolaf.edu/gca/. Accesos: 18 de Abril (2013)

EPA. Health Effects Assessment Summary Tables (HEAST) (en línea). US Environmental Protection Agency, Washington, DC, USA (2008). http://cfpub.epa.gov/ncea/cfm/recordisplay.cfm?deid=2877. Acceso: 18 de Abril (2013).

EPA, Estimation Programs Interface Suite ${ }^{\mathrm{TM}}$ for Microsoft ${ }^{\circ}$ Windows, $v 4.11$ (en línea). US Environmental Protection Agency, Washington, DC, USA (2012). http://www.epa.gov/oppt/exposure/pubs/episuite.htm. Acceso: 18 de Abril (2013).

EPA. Integrated Risk Information System (IRIS) (en línea). US Environmental Protection Agency, Washington, DC, USA (2013). http://www.epa.gov/iris/index.html. Acceso: 18 de Abril (2013)

García-Serna, J., L. Pérez-Barrigón y M.J. Cocero, New trends for design toward sustainability in chemical engineering: Green engineering, Chemical Engineering Journal, 133(1-3), 7-30 (2007).

Gutiérrez-Martín, F. y M.F. Dahab, Issues of sustainability and pollution prevention in environmental engineering education, Water Science and Technology, 38(11), 271-278 (1998).

Gutiérrez-Martín, F. y S.H. Hüttenhain, Environmental education; new paradigms and engineering syllabus, J. Cleaner Production, 11(3), 247-251 (2003).

Gutiérrez, F., M.A. Sanchiz, M.T. Hernández y E. Atanes, Chemical engineering: new paradigms and environmental syllabus, Actas del 4th European Congress of Chemical Engineering, P-1.1-012, Granada, España, 21 a 25 de Septiembre (2003).

Gutiérrez, F. y A. Quirce, Environmental conscious education for Chemical Engineers, In Engineering Education in Sustainable Development (EESD 2004), Eds: D. Ferrer-Balas, K.F. Mulder, J. Bruno, R. Sans, pp. 132 CIMNE and UPC, Barcelona, España (2004).

Gutiérrez, F. y M.T. Domínguez, Información y control de riesgos químicos en el nuevo reglamento REACH, Ingeniería Química, 435, 168-170 (2006).

Gutiérrez-Martín, F., P. Díaz Fernández-Zapata, M.A. Sanchiz-Rocha y M.T. Hernández-Antolín, Nuevos paradigmas y métodos para la educación ambiental en la ingeniería: producción limpia, ecología industrial y desarrollo sostenible, Cuadernos de Innovación Educativa en las Enseñanzas Técnicas, 1(2), 11-20 (2007).

Gutiérrez-Martín, F., M.T. Hernández-Antolín, M.A. Sanchiz-Rocha y P. Díaz Fernandez-Zapata, Aplicación de Software Libre a la Evaluación de Contaminantes en el Medio Ambiente, Actas del 15을 Congreso de Innovación Educativa en Enseñanzas Técnicas, 705-714, Valladolid, España, 18 a 20 de Septiembre (2007).

Howard P. y W. Meylan, Assessing environmental fate and exposure, Chemical Engineering, 3, 91-96 (2001). 
ISTAS, ECO informas (en línea). Instituto Sindical de Trabajo, Ambiente y Salud, CCOO y Fondo Social Europeo (2006). http://www.istas.net/ecoinformas08/web/. Acceso: 18 de Abril (2013).

Lancaster, M., Principles of Sustainability and Green Chemistry, In Handbook of Green Chemistry \& Technology by J. Clark \& D. Macquarrie, pp 10-26 Blackwell Pub., Oxford, UK (2002).

Lemkowitz S.M., B.H. Bibo, G.H. Lameris y B.A. Bonet, From small scale, short term to large scale, long term: integrating sustainability into engineering education, European Journal of Engineering Education, 4, 353-386 (1996).

Mackay, D. y S. Paterson, Evaluating the multimedia fate of organic chemicals: a level III fugacity model, Environmental Science \& Technology, 25(3), 427-436 (1991).

Mackay, D., Multimedia Environmental Models: The Fugacity Approach, 2ª edición, 1-261. Lewis Publishers, CRC Press, Boca Raton, FL, USA (2001).

Mulder, K.F., Technology for Sustainable Development, In Sustainable Development for Engineers by K. Mulder, Delft University of Technology, pp 251-277 Greenleaf Pub., Sheffield, UK (2006).

Nieto-Márquez, A., A. Cambra y F. Gutiérrez-Martín, Evaluación de impactos ambientales bióticos en la industria química mediante uso de software libre, Actas del I Congreso de Innovación Docente en Ingeniería Química, 31, Granada, España, 26 a 27 de Enero (2012).

Nieto-Márquez, A., E. Atanes-Sánchez y F. Gutiérrez-Martín, Free software for evaluation of biotic environmental impacts in chemical process industries (CPIs), ANQUE International Congress of Chemical Engineering, T15-003, Sevilla, España, 24 a 27 de Junio (2012).

NIHS. National Institute of Health Sciences (en línea). Kamiyoga 1-18-1, Setagaya-ku, Tokyo 158-8501, JAPAN (2006). http://www.nihs.go.jp/english/index.html. Acceso: 18 de Abril (2013).

NTP. National Toxicology Program (en línea). National Institute of Environmental Health Sciences, NC, USA (2013). http://ntp-server.niehs.nih.gov/. Acceso: 18 de Abril (2013).

OCW. Producción limpia, ecología industrial y desarrollo sostenible (en línea). Universidad Politécnica de Madrid (2006). http://ocw.upm.es/ingenieria-quimica/. Acceso: 18 de Abril (2013).

OSHA. Occupational, Safety \& Health Administration (en línea). US Department of Labor, Washington, DC, USA (2013). http://www.osha.gov/as/opa/spanish/index.html. Acceso: 18 de Abril (2013).

Rosenbaum, R.K., M. Margni y O. Jolliet, A flexible matrix algebra framework for the multimedia multipathway modeling of emission to impacts, Environment International, 33(5), 624-634 (2007).

Serrano, J.E. y P.S. Narváez, Uso de software libre para el desarrollo de contenidos educativos, Formación Universitaria, 3(6), 41-50 (2010). 